\title{
Comparison of Efficiencies of Neurological Physical Examination, Neurothesiometer and PainDETECT Questionnaire in Diagnosing Diabetic Neuropathy
}

\author{
Hajrah Ahsan ${ }^{1}$, Talha Kareem², Salma Tanveer ${ }^{1}$, Muhammad Farrukh Aftab ${ }^{2}$, Bahawal Zeb ${ }^{3}$ and Ahmad
} Kabir $^{1}$

1. Department of General Medicine, Nishtar Hospital, Multan, Pakistan

2. Department of General Surgery, Nishtar

Hospital, Multan, Pakistan

3. Department of General Medicine, Combined

Military Hospital,

Multan, Pakistan

*Correspondence: farrukhaf2001@yahoo.co $\mathrm{m}$

Keywords: Diabetic neuropathy, MNSI, neurothesiometer, PainDETECT

doi:

10.5281/zenodo.3610652

Supplementary and additional files (if any) for all TIJFS publications can be found online at www.tijfs.com

Submitted: July 30, 2019 Accepted: August 26, 2019

Published Online: September 9, 2019

How to cite this: Authors. 2020.

Manuscript Title. Int J Front Sci, 4(1), Pages.

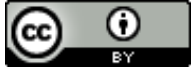

This article is open access under terms of Creative Commons Attribution License 4.0. which permits unrestricted use distribution and reproduction in any medium provided the original work is cited properly.

\section{Significance: \\ Diabetic neuropathy is a very common condition prevalent is Diabetics. Neuropathy is present in most of the patients affected by diabetes and is major cause of their morbidity. The pathogenesis of diabetic neuropathy has been attributed to dysfunction of metabolic pathways by chronic hyperglycemia. This hyperglycemia leads in reduced ability of tissues to detoxify free radicals. Current study found efficiencies of diabetic neuropathy diagnosis techniques.}

\section{ABSTRACT}

Objective: To compare the efficacies of neurological physical examination, neurothesiometer and PainDETECT questionnaire in diagnosing diabetic neuropathy.

Study Design: Prospective cross-sectional

Place and Duration of Study: Department of General Medicine, Nishtar Hospital Multan, Pakistan from $1^{\text {st }}$ December 2018 to $10^{\text {th }}$ March 2019.

Materials and Methods: One hundred and four patients of both type 1 and type 2 diabetes visiting the outdoor department were included in this study. They were assessed by lab results of glycosylated hemoglobin, fasting and random blood sugar levels and neurological physical examination.

Results: The physical examination with Michigan Neuropathy Screening instrument showed that around 29 of the patients were having established neuropathy. The PainDETECT questionnaire on the other hand showed about 42 patients having a definitive neuropathy while the neurothesiometer showed that 79 of the total patients had varying degrees of neuropathy. Conclusion: The neurothesiometer is a better diagnostic tool for diagnosing diabetic neuropathy in patients.

\section{Introduction}

Diabetic neuropathy is a very common condition prevalent is Diabetics. Neuropathy is present in most of the patients affected by diabetes and is major cause of their morbidity. (1) The pathogenesis of diabetic neuropathy has been attributed to the dysfunction of the metabolic pathways by the chronic hyperglycemia. It is postulated that this hyperglycemia leads in the reduced ability of tissues to detoxify free radicals. This oxidative stress can in turn lead to peripheral neuropathy. (2) The polyol pathway was found to be a major determinant of this oxidative stress. Data suggest that the increase in the influx of the substrate in this pathway is the primary pathogenic factor in diabetic neuropathy. (3)

Studies have demonstrated that reduced nerve perfusion and hypoxia of endoneurium has also played a pivotal role in development of diabetic neuropathy. Biopsy was taken from patients suffering from mild to moderate neuropathy and it showed features like basement membrane thickening, pericyte degeneration and endothelial cell hyperplasia. $(4,5)$

\section{Materials and Methods:}

This prospective cross-sectional study was conducted at Department of General Medicine, Nishtar Hospital Multan, Pakistan from $1^{\text {st }}$ December 2018 to $10^{\text {th }}$ March 2019. Patients with both type 1 and 2 diabetes were included. Hypertension was defined as having blood pressure more than $140 / 90 \mathrm{~mm} \mathrm{Hg}$. A fasting cholesterol level of more than $200 \mathrm{mg} / \mathrm{dl}$ was labelled as hypercholesterolemia. 104 consecutive patients presenting in outdoor department, fulfilling the inclusion criteria were included in the study. Informed consent was taken from all patients. A positive urine dipstick was considered as overt diabetic nephropathy in the absence of any other causes of proteinuria. Serum urea and creatinine were checked for any renal disorder. All the patients included in this research had their random blood sugar level monitored using an On $\mathrm{Call}^{\circledR}$ EZ II glucometer. Patients had their HbA1c (glycosylated hemoglobin) measured using immunoturbidimetric by hospital's inhouse Laboratory. All the patients coming to the OPD had their assessment of palpation of dorsalis pedis and posterior tibial arteries was made, and they were characterized as being "present" or "absent". The diabetic neuropathy in these patients was assessed using the PainDETECT questionnaire for the neuropathic pain along with the physical examination that was scored using the MNSI 
criteria and the neurothesiometer by health care professional.

PainDETECT is an easy questionnaire that is designed to differentiate the nociceptive and the neuropathic pain. It is designed so the results solely rely on the characteristic clinical neuropathic pain. Each parameter is graded as to obtain an overall idea of the extent of the neuropathy. Michigan neuropathy screening instrument (MNSI) was used for screening on basis of physical examination. (6) On physical assessment the first step was to observe if the appearance of the feet was normal. A keen observation was made regarding any deformity, callus, infection, fissure or any other anomaly that may compel as to label the appearance as abnormal. 0 was the score assigned to the normal feet and if there was anything that did not fit the norm the score was 1 (for each foot). If there was no ulceration, the score was 0 but a score of 1 was assigned for an ulcer found on each foot. A reflex hammer was used to access the ankle reflex. Normal ankle reflex was given 0 score, diminished reflex 0.5 and 1 was allotted to an absent ankle reflex. MNSI also requires the perception of vibration to be evaluated. In our study, we used a $128 \mathrm{~Hz}$ vibration tuning fork to aid in our cause. Tuning fork was placed at the ball of the big toe. If the patient could perceive it then medial malleolus was tested. A better perception of vibration sense in the medial malleolus was given 0.5 score. If the perception was equal in both, the vibration sense was ascertained to be normal and given the score 0 . Failure to perceive vibration sense in big toe was given a score of 1 in each foot. To test the presence of pressure sensation in the sole of the foot $10 \mathrm{~g}$ monofilaments (also called as Semmes Weinstein monofilaments) were used. The heads of $1^{\text {st }}, 3^{\text {rd }}, 5^{\text {th }}$ metatarsal heads and plantar surface of distal hallux. The technique for testing is that the patient should have his/her eyes closed and the monofilament should be applied to the designated sites until it buckles. The buckling shows that a pressure of $10 \mathrm{~g}$ has been applied. In this research, disposable monofilaments by Booth and Young were used.

The vibration perception threshold can be semiquantitatively assessed using a neurothesiometer. It can be assessed by asking the patient to lie down on a couch. The stylus of the instrument is then placed over the pulp of the hallux. The amplitude of the vibration is increased until the patient can perceive the vibration. The resultant value obtained is called as the VPT (vibration perception threshold). Initially the process is done over the proximal site and then the readings are repeated over different points of pain on the sole of the foot. The results are variable for different neurothesiometers used. The one used in our research had a normal threshold at 1-10 volts. Anything between 11-15 was deemed as mild neuropathy. 16-20 volts depicted a moderate one and anything above 20 was said to be severe neuropathy. A severe neuropathy has been strongly associated with the development of subsequent foot ulceration. The data was analyzed using SPSS-20.

\section{Results}

HbA1C levels of the patients in this research varied from $7-14 \%$ with an average of $9.1 \%$. While assessing the effectiveness of various methods for diabetic neuropathy, three variables were primarily put into use. The duration for which the patients had diabetes, the type of diabetes they were having and the treatment they were currently upon (Table 1 ).

MNSI suggests that score of $\geq 2.5$ is considered the standard for diabetic neuropathy. Twenty-nine of the patients in the research were suffering from diabetic neuropathy according to the criteria of MNSI. Most of the patients in this category had comorbidity as well. Nine of the patients were hyperlipidemic with 8 taking medications for it. 11 were hypertensive and had a reduced renal function and 13 had some degree of diabetic retinopathy (Table 2 ).

The PainDETECT questionnaire helped us in identifying whether the pain, that the patients in our research had was of nociceptive or neuropathic in origin. Forty-two of the patients were screened as having neuropathic pain. Twenty-four had them labeled as nociceptive while the result was unclear in about 38 of the patients (Fig. 1).

A score of greater than 18 (19-38) is considered as neuropathic pain. The average duration of diabetes for individuals in this group was 13 years. Of the 42 patients who were screened to have a neuropathic pain, 21 of them were hypertensive with 16 taking regular medications for it. 4 were hyperlipidemic and 17 had some degree of retinopathy (Table 3 ).

Among the 104 patients in our research, 79 had some sort of neuropathy. Only 26 had the vibration threshold below 10 volts. 32 had mild neuropathy, 26 had moderate while 20 patients suffered from severe neuropathy. Of the 29 patients deemed neuropathic using the MNSI 27 were also labeled as neuropathic using neurothesiometer. Similarly, all the 40 neuropathic pain cases in the PainDETECT group were also tested positive on neurothesiometer (Table 4).

The results from neurothesiometer not only covered majority of the individuals who had co morbidities but there were a good number of individuals who were asymptomatic but had an increased vibration perception threshold (Fig. 2). 
Table 1: Demographic information of the patients $(n=104)$

\begin{tabular}{|l|c|}
\hline Age & $53.92 \pm 13.158$ \\
\hline Male/Female & $42 / 62$ \\
\hline DM disease length (years) & $10.45 \pm 6.753$ \\
\hline Type 1/ Type 2 & $24 / 80$ \\
\hline BMI $\left(\mathrm{kg} / \mathrm{m}^{2}\right)$ & 32.6 \\
\hline Patients on oral hypoglycemic & 22 \\
\hline Patients taking insulin only & 31 \\
\hline Patients taking both regimens & 51 \\
\hline
\end{tabular}

Table 2: Number of positive patients $(n=29)$

\begin{tabular}{|l|c|}
\hline Mean score of total subjects & $1.6058 \pm 1.14203$ \\
\hline $\begin{array}{l}\text { Mean duration of DM } \\
\text { (years) }\end{array}$ & $18.69 \pm 5.373$ \\
\hline Type 1/ Type 2 & $7 / 22$ \\
\hline Oral hypoglycemic only & 4 \\
\hline Insulin only & 6 \\
\hline $\begin{array}{l}\text { Oral hypoglycemic and } \\
\text { insulin }\end{array}$ & 19 \\
\hline
\end{tabular}

Table 3: Neurologic pain $(n=42)$

\begin{tabular}{|l|c|}
\hline Mean score & $17.1 \pm 6.9$ \\
\hline Mean duration of diabetes & $15.14 \pm 6.580$ \\
\hline Type 1/ Type 2 & $5 / 37$ \\
\hline Oral hypoglycemics only & 8 \\
\hline Insulin only & 8 \\
\hline $\begin{array}{l}\text { Oral hypoglycemics and } \\
\text { Insulin }\end{array}$ & 26 \\
\hline
\end{tabular}

Table 4: Number of neuropathic patients $(n=79)$

\begin{tabular}{|l|c|}
\hline Mean duration (years) & $11.67 \pm 6.978$ \\
\hline Type 1/Type 2 & $13 / 65$ \\
\hline Oral hypoglycemic only & 14 \\
\hline Insulin only & 20 \\
\hline $\begin{array}{l}\text { Oral hypoglycemics and } \\
\text { insulin }\end{array}$ & 44 \\
\hline
\end{tabular}

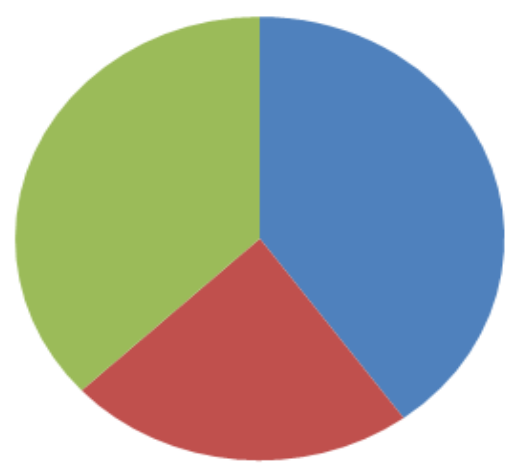

- Neuropathic Nociceptive Unclear

Fig. 1: PainDETECT screening

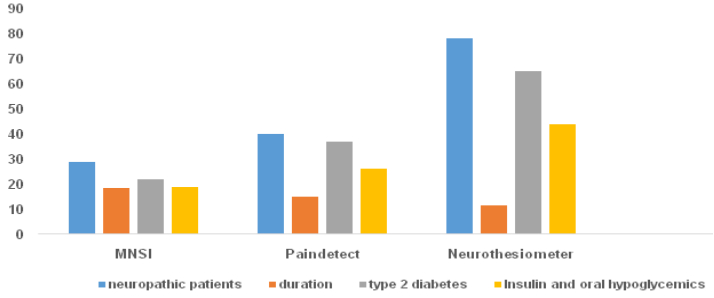

Fig. 2: Comparison of neuropathic assessment

\section{Discussion}

Diabetic neuropathy refers to a broad term encompassing both the peripheral and the autonomic neuropathies. Among the peripheral neuropathies, distal symmetric neuropathy is the most common form. Association of neuropathy with glycemic control and duration has been well established by multiple studies $(1,7)$ emphasizing the importance of early detection and strict glycemic control. There are documented suggestions that in case of type 1 diabetes, glycemic control has been fundamental in controlling/prolonging the neuropathy free interval as opposed to type 2 diabetes that not only requires tight glycemic control but the lipid profile, lifestyle habits and blood pressure all have a vital role to play. (8) There is evidence of obesity and hypertriglyceridemia being individual factors in the early onset of neuropathy, independent from tight glycemic control. (9) When considering the possibility of diabetic neuropathy, vascular risk factors have their own specific role to play as evident by the EURODIAB prospective complications study. (10)

In our study, we evaluated 104 patients, suffering from both type 1 and type 2 diabetes. The focus, however, was their development of neuropathy. Being a debilitating result of diabetes, it is crucial that there should be a system for early detection of neuropathy. Here we employed three different methods, MNSI, 
PainDETECT and neurothesiometer, in order to assess their efficiency in detecting neuropathy in diabetic patients.

MNSI has been used as a screening tool for diabetic neuropathy. It includes two components, a 15-item questionnaire and a physical examination involving lower limb. MNSI has been regarded as an easily workable and elementary screening tool to be used in clinical examination for diagnosis of diabetic neuropathy. (11) MNSI score was $\geq 2.5$ in 29 cases labelling them as neuropathic. In a study in Turkey, they assessed patients on the number of questions they answered on the MNSI questionnaire. (6) They employed a total of 106 patients and 34 of them had positive history regarding neuropathy. 24 of their patients gave a positive response for $\geq 7$ questions and among 72 not diagnosed with diabetic neuropathy, 28 had a positive response for $\geq 7$ questions. This brings us to our research. Of the 29 who were having a $\geq 2.5$ score on the MNSI 22 gave an affirmative answer to $\geq 7$ questions. Of the remaining having a reduced score on MNSI, there were 21 cases where the answer was same implying that diagnosing neuropathy based on symptoms can be evasive.

Screening questionnaires like PainDETECT are convenient to use in daily practice but a study conducted implies that the major characteristics in this questionnaire, like numbness and burning sensations, are subjective and so therefore it is not sufficient to point out the numerous sensory loses attributed to neuropathy. (12) This questionnaire has though afforded the benefit of segregating patients based on nociceptive and neuropathic pain and at the same time helped clinicians to identify if a particular patient is to be referred to a specialist for pain management. An extensive study has shown PD-Q being used for chronic pain and it is being assumed that in the future it can be employed to partition the individual sensory profiles aiding the medical personnel to personify the pain treatment. (13) Another important benefit of this questionnaire is that it does not require any sort of clinical examination and anyone, even the patient themselves, can grade them. This will help them understand as to when they need professional help.

Vibration sense is one of the earliest ones to be lost when the diabetic neuropathy sets in. This along with pain and numbness start distally in fingers and toes and then extending proximally producing the classic 'Glove and Stocking' deformity. In our research we used a portable neurothesiometer, that was easy to use and had a lower threshold than tuning forks to determine the vibration sense in patients. VPT determined by neurothesiometer labeled 78 patients in our research as suffering from diabetic neuropathy that was almost twice what the other methods had detected. A study has shown that VPT determined with neurothesiometer is relatively constant facilitating us to use this as a tool in clinical trials. $(14,15) \mathrm{A}$ possibility is that the neurothesiometer may have over predicted the number of neuropathic patients. We compared the ones who were 'mildly' suffering from the disease according to the neurothesiometer. Of the 32 people in this category, only 1 was diagnosed as having neuropathy by MNSI while PainDETECT had 4 in the unclear category with only 2 as neuropathic. Moreover, neurothesiometer also had an edge as it detected a few of those patients to be neuropathic who had no other comorbidities making it a useful clinical tool in early screening of Diabetic neuropathy.

\section{Conclusion}

Neurothesiometer is far superior than other two methods in diagnosing early neuropathy and preventing its complications.

Conflict of Interest: This study has no conflict of interest to declare by any author.

Disclosure: None

Human and Animal Rights: No rights violated

\section{References}

1. Dyck PJ, Davies JL, Wilson DM, Service FJ, Melton LJ, 3rd, O'Brien PC. Risk factors for severity of diabetic polyneuropathy: intensive longitudinal assessment of the Rochester Diabetic Neuropathy Study cohort. Diabetes Care 1999;22(9):1479-86.

2. Vincent AM, Russell JW, Low P, Feldman EL. Oxidative stress in the pathogenesis of diabetic neuropathy. Endocrine Rev 2004;25(4):612-28.

3. Oates PJ. Polyol pathway and diabetic peripheral neuropathy. Int Rev Neurobiol 2002;50:325-92.

4. Cameron NE, Eaton S, Cotter MA, Tesfaye S. Vascular factors and metabolic interactions in the pathogenesis of diabetic neuropathy. Diabetologia 2001;44(11):1973-88.

5. Yagihashi S, Yamagishi S-I, Wada R. Pathology and pathogenetic mechanisms of diabetic neuropathy: correlation with clinical signs and symptoms. Diabetes Res Clin Prac 2007;77(3):S184-S9.

6. Mete T, Aydin Y, Saka M, Cinar Yavuz H, Bilen S, Yalcin $\mathrm{Y}$, et al. Comparison of efficiencies of michigan neuropathy screening instrument, neurothesiometer, and electromyography for diagnosis of diabetic neuropathy. Int J Endocrinol 2013;2013.

7. Nisar MU, Asad A, Waqas A, Ali N, Nisar A, Qayyum MA, et al. Association of Diabetic Neuropathy with Duration of Type 2 Diabetes and Glycemic Control. Cureus 2015;7(8):e302-e.

8. Callaghan BC, Hur J, Feldman EL. Diabetic neuropathy: one disease or two? Curr Opin Neurol 2012;25(5):536.

9. Smith AG, Singleton JR. Obesity and hyperlipidemia are risk factors for early diabetic neuropathy. J Diabetes Complications 2013;27(5):436-42.

10. Tesfaye S, Chaturvedi N, Eaton SE, Ward JD, Manes C, Ionescu-Tirgoviste $\mathrm{C}$, et al. Vascular risk factors and diabetic neuropathy. N Engl J Med 2005;352(4):341-50.

11. Herman W, Pop-Busui R, Braffett B, Martin C, Cleary P, Albers J, et al. Use of the Michigan Neuropathy Screening Instrument 
as a measure of distal symmetrical peripheral neuropathy in Type 1 diabetes: results from the Diabetes Control and Complication Trial/Epidemiology of Diabetes Interventions and Complications. Diabetic Med 2012;29(7):937-44.

12. Vollert J, Kramer M, Barroso A, Freynhagen R, Haanpaa M, Hansson P, et al. Symptom profiles in the PainDETECT Questionnaire in patients with peripheral neuropathic pain stratified according to sensory loss in quantitative sensory testing. Pain 2016;157(8):1810-8.
13. Freynhagen R, Tölle TR, Gockel U, Baron R. The PainDETECT project-far more than a screening tool on neuropathic pain. Curr Med Res Opin 2016;32(6):1033-57.

14. Bril V, Kojic J, Ngo M, Clark K. Comparison of a neurothesiometer and vibration in measuring vibration perception thresholds and relationship to nerve conduction studies. Diabetes care 1997;20(9):1360-2.

15. Kincaid JC, Price KL, Jimenez MC, Skljarevski V. Correlation of vibratory quantitative sensory testing and nerve conduction studies in patients with diabetes. Muscle Nerve 2007;36(6):821-7. 